REVISTA PROYECCIONES N ${ }^{\circ} 7: 69-96$

JUNIO 1984 - I.S.S.N. 0716-0917

\title{
UN ALGORITMO CONVERGENTE PARA LOCALIZAR TODAS LAS RAÍCES DE UNA FUNCIÓN LIPSCHITZ-CONTINUA
}

GABRIEL GATICA P. $(*)$

RESUMEN .

Se modifica un método de SUKHAREV [5] y se propone un nuevo alforitmo Block-secuencial para localizar todas las raíces de una función Lipschitz-continua. Se demuestra la convergencia de esta estrategia y se dan algunos ejemplos numéricos.

(*) Departamento de Matemática, Facultad de Ciencias, Universidad de Con cepción. 


\section{INTRODUCCION.}

Se considera una función $f:[a, b] \rightarrow \mathbb{R}$ y se define el conJUNTO SOLUCION COMO:

$$
R_{f}[a, b]=\{x \in[a, b] / f(x)=0\}
$$

Se dice que L $\underline{c}[a, b]$ es una LOCALIZACION DEL CONJUNTO SOLU CION si $R_{f}[a, b] \subseteq \underline{c} L$. La idea común a cada uno de los métodos de localización de raíces es construir una clase de funciones $\delta$ que contenga a $f, y$ dos funciones $\mathrm{F}^{-} \mathrm{y} \mathrm{F}^{+}$, llamadas MINORANTE $\mathrm{Y}$ MAYORANTE, respecti vamente, tales que:

$$
F^{-}(x) \leqslant g(x) \leqslant F^{+}(x) ; \forall x \in[a, b], \forall g \in \delta .
$$

La obtención de $\delta, \mathrm{F}^{-} \mathrm{y} \mathrm{F}^{+}$se logra mediante evaluaciones de $\mathrm{f}$ en un número finito de puntos de $[a, b], y$ en base a alguna información global acerca de las propiedades de la función $f . \mathrm{F}^{-}$y $\mathrm{F}^{+}$constituyen la llamada ENVOLVENTE de $\delta$ cuando ocurre $\mathrm{F}^{-}, \mathrm{F}^{+} \mathrm{e} \delta, \mathrm{y}$ las raíces de ella, que se suponen fáciles de determinar, permiten calcular una localización de $R_{f}[a, b]$.

Se puede clasificar entonces los métodos que buscan las raíces de una función de valores reales f sobre un intervalo $[a, b]$ de acuerdo a la clase de funciones a la cual pertenece $\mathrm{f}$ y a la estrategia usada para elegir los puntos de evaluación. Si se da una elección a priori de ellos, se dice que el método usa una ESTRATEGIA PASIVA. De otro modo, si la elección de cada punto depende de la ubicación de los anterio res y de los valores de $f$ ya obtenidos, se dice que el método usa una ESTRATEGIA SECUENCIAL.

Como ya se ha dicho, uno de los factores que define un método de búsqueda es la clase de funciones a la cual se aplica. Es lógico que mientras más hipótesis se hagan sobre la función, mejores métodos de localización se pueden crear. Es de esperar entonces, que con restriccio- 
nes como monotonía o unicidad se desarrollen técnicas muy eficientes, en tanto que si se consideran clases más generales de funciones, los métodos obtenidos sean complicados e ineficientes. Por lo tanto, desde este punto de vista se puede distinguir en principio dos grandes clases de mé todos de acuerdo a si se requiere o no una hipótesis de unicidad para las raíces de $\mathrm{f}$.

Los métodos diseñados para funciones con una sola raíz (CHERNOUSKO [3], MICCHELLI Y MIRANKER [4] aunque simples, son de aplicación limitada ya que la hipótesis es muy restrictiva y en la práctica es difícil verificarla. Desafortunadamente, y es lo que motivó en gran par te este artículo, la literatura es muy escasa cuando se buscan métodos que no suponen unicidad y sólo se pueden mencionar los métodos de BAsso [2] y SUKHAREV [5]. Nótese, eso sí, que en [2] se desarrollan técnicas para encontrar todas las raíces de la función, en cambio, SUKHAREv sólo localiza una de ellas.

Una limitación práctica importante de los métodos de localización de raíces es la dificultad para obtener la información necesaria. Por ejemplo, para las funciones Lipschitz-continuas, que se abordarán aquí, se requiere conocer una cota del supremo esencial de $\left|f^{\prime}\right|$ lo cual constituye, a veces, un problema del mismo tipo y grado de dificultad que el cálculo de las raíces de una función.

En este artículo se considera una función

$\mathrm{f} \in \operatorname{Lip}[\mathrm{a}, \mathrm{b}]_{\mathrm{K}}=\{\mathrm{g}:[\mathrm{a}, \mathrm{b}] \rightarrow \mathbb{R} /|\mathrm{g}(\mathrm{x})-\mathrm{g}(\mathrm{y})| \leqslant \mathrm{K}|\mathrm{x}-\mathrm{y}| ; \forall \mathrm{x}, \mathrm{y} \in[\mathrm{a}, \mathrm{b}]\}$, un conjunto de puntos $\left\{\mathrm{x}_{1}, \ldots, \mathrm{x}_{n}\right\} \subseteq[\mathrm{a}, \mathrm{b}]$ y se reconstruye la envolvente dada por SUKHAREV [5] para la clase de funciones:

$\delta\left(f ; x_{1}, \ldots, x_{n}\right)=\left\{p \in \operatorname{Lip}[a, b]_{K} / \varphi\left(x_{i}\right)=f\left(x_{i}\right) ; \quad V i=\overline{1, n}\right\}$

Se calcula además las raíces de esta envolvente y los valores estremos del mayorante y minorante que ella define. El método de Sukharev es modificado en la sección 2 con el fin de localizar todas las raíces de f. Para tal efecto se construye una sucesión de mayorantes y minoran- 
tes que convergen puntualmente a en $[a, b]$, y se prueba que la intersec ción de las localizaciones obtenidas es igual a $R_{f}[a, b]$. Finalmente en sección 3 se muestran algunos ensayos numéricos.

\section{1. eNVOLVENTE DE LA CLASE $\delta\left(f_{;} x_{1}, x_{2}, \ldots, x_{n}\right)$}

Dado $\mathrm{K}>0$, se considera la clase de funciones:

$\operatorname{Lip}[a, b]_{K}=\{g:[a, b] \rightarrow \mathbb{R} /|g(x)-g(y)| \leqslant K|x-y| ; \forall x, y \in[a, b]\}$

Para toda función $g \in \operatorname{Lip}[a, b]_{K}$ se define:

$\delta(g)=\operatorname{Lip}[a, b]_{K}$

$\delta\left(g ; x_{1}\right)=\left\{\emptyset \in \delta(g) / \varphi\left(x_{1}\right)=g\left(x_{1}\right)\right\}$

$\delta\left(g ; x_{1}, \ldots, x_{n}\right)=\left\{b \in \delta\left(g ; x_{1}, \ldots, x_{n-1}\right) / \phi\left(x_{n}\right)=g\left(x_{n}\right)\right\}$

$R_{g}[a, b]=\{x \in[a, b] / g(x)=0\}$

1.1. Envolvente de $\mathrm{f} \in \operatorname{Lip}[\mathrm{a}, \mathrm{b}]_{\mathrm{K}^{*}}$

Se considera ahora una función $f \in \operatorname{Lip}[a, b]_{K}$ y se supone que $f$ se ha evaluado en $n$ puntos $x_{1}, x_{2}, \ldots, x_{n}$ de $[a, b]$ obteniendo:

$$
y_{j}=f\left(x_{j}\right) \quad ; \quad \forall j \in\{1, \ldots, n\}
$$

Sea:

$$
\left.\begin{array}{l}
F_{n}^{-}(x)=\max _{j \in\{1, \ldots, n\}}\left\{y_{j}-k\left|x-x_{j}\right|\right\} \\
F_{n}^{+}(x)=\min _{j \in\{1, \ldots, n\}}\left\{y_{j}+k\left|x-x_{j}\right|\right\}
\end{array}\right\}
$$

entonces, para toda $b \in \delta\left(f_{;} x_{1}, \ldots, x_{n}\right.$ ) resulta:

$$
\mathrm{F}_{\mathrm{n}}^{-}(\mathrm{x}) \leqslant \varphi(\mathrm{x}) \leqslant \mathrm{F}_{\mathrm{n}}^{+}(\mathrm{x}) ; \forall \mathrm{x} \in[\mathrm{a}, \mathrm{b}]
$$


Se observa de aquí que $\mathrm{F}_{\mathrm{n}}^{-} \mathrm{y} \mathrm{F}_{\mathrm{n}}^{+}$son MINORANTE y MAYORANTE, y constituyen la envolvente de la clase de funciones $\delta\left(f ; x_{1}, \ldots, x_{n}\right.$ ).

Sea ahora el conjunto $\left\{p_{1}, \ldots, p_{n}\right\}$ tal que $\left\{\mathrm{p}_{1}, \ldots, \mathrm{p}_{\mathrm{n}}\right\}=\left\{\mathrm{x}_{1}, \ldots, \mathrm{x}_{\mathrm{n}}\right\}$ y $\mathrm{p}_{1}<\mathrm{p}_{2}<\ldots<\mathrm{p}_{\mathrm{n}}$.

Sean además $z_{i}=f\left(p_{i}\right), \forall i \in\{1, \ldots, n\}$. Luego (7) puede reemplazarse por:

$$
\begin{aligned}
& F_{n}^{-}(x)=\max _{i \in\{1, \ldots, n\}}\left\{z_{i}-k\left|x-p_{i}\right|\right\} \\
& F_{n}^{+}(x)=\min _{i \in\{1, \ldots, n\}}\left\{z_{i}+k\left|x-p_{i}\right|\right\}
\end{aligned}
$$

(Ver Figura 1).

Sean $\mathrm{F}_{\mathrm{n}, \mathrm{i}}^{-} \mathrm{y} \mathrm{F}_{\mathrm{n}, \mathrm{i}}^{+}$las restricciones de $\mathrm{F}_{\mathrm{n}}^{-} \mathrm{y} \mathrm{F}_{\mathrm{n}}^{+}$al intervalo $\left[p_{i}, p_{i+1}\right]$. Entonces, de (9) resulta:

$$
\begin{aligned}
& \mathrm{F}_{\mathrm{n}, i}^{-}(\mathrm{x})=\max \left\{\mathrm{z}_{i}-\mathrm{K}\left|\mathrm{x}-\mathrm{p}_{\mathrm{i}}\right| ; \mathrm{z}_{i+1}-\mathrm{K}\left|\mathrm{x}-\mathrm{p}_{\mathrm{i}+1}\right|\right\} \\
& \mathrm{F}_{\mathrm{n}, \mathrm{i}}^{+}(\mathrm{x})=\min \left\{\mathrm{z}_{i}+\mathrm{K}\left|\mathrm{x}-\mathrm{p}_{\mathrm{i}}\right| ; \mathrm{z}_{\mathrm{i}+1}+\mathrm{K}\left|\mathrm{x}-\mathrm{p}_{\mathrm{i}+1}\right|\right\} \\
& \forall \mathrm{x} \in\left[\mathrm{p}_{i}, \mathrm{p}_{\mathrm{i}+1}\right], \quad \forall i \in\{1, \ldots, \mathrm{n}-1\}
\end{aligned}
$$

(Ver Figura 2).

Por otro lado, si $\mathrm{F}_{\mathrm{n}, \mathrm{o}}^{-} \mathrm{y} \mathrm{F}_{\mathrm{n}, \mathrm{o}}^{+}$(respectivamente $\mathrm{F}_{\mathrm{n}, \mathrm{n}}^{-} \mathrm{Y} \mathrm{F}_{\mathrm{n}, \mathrm{n}}^{+}$son las restricciones de $\mathrm{F}_{\mathrm{n}}^{-} \mathrm{y} \mathrm{F}_{\mathrm{n}}^{+{ }^{+}}$al intervalo $\left[\mathrm{a}, \mathrm{p}_{1}\right]$ (respectivamente $\left.\left[p_{n, b}\right]\right)$, entonces de (9) se obtiene: 
74

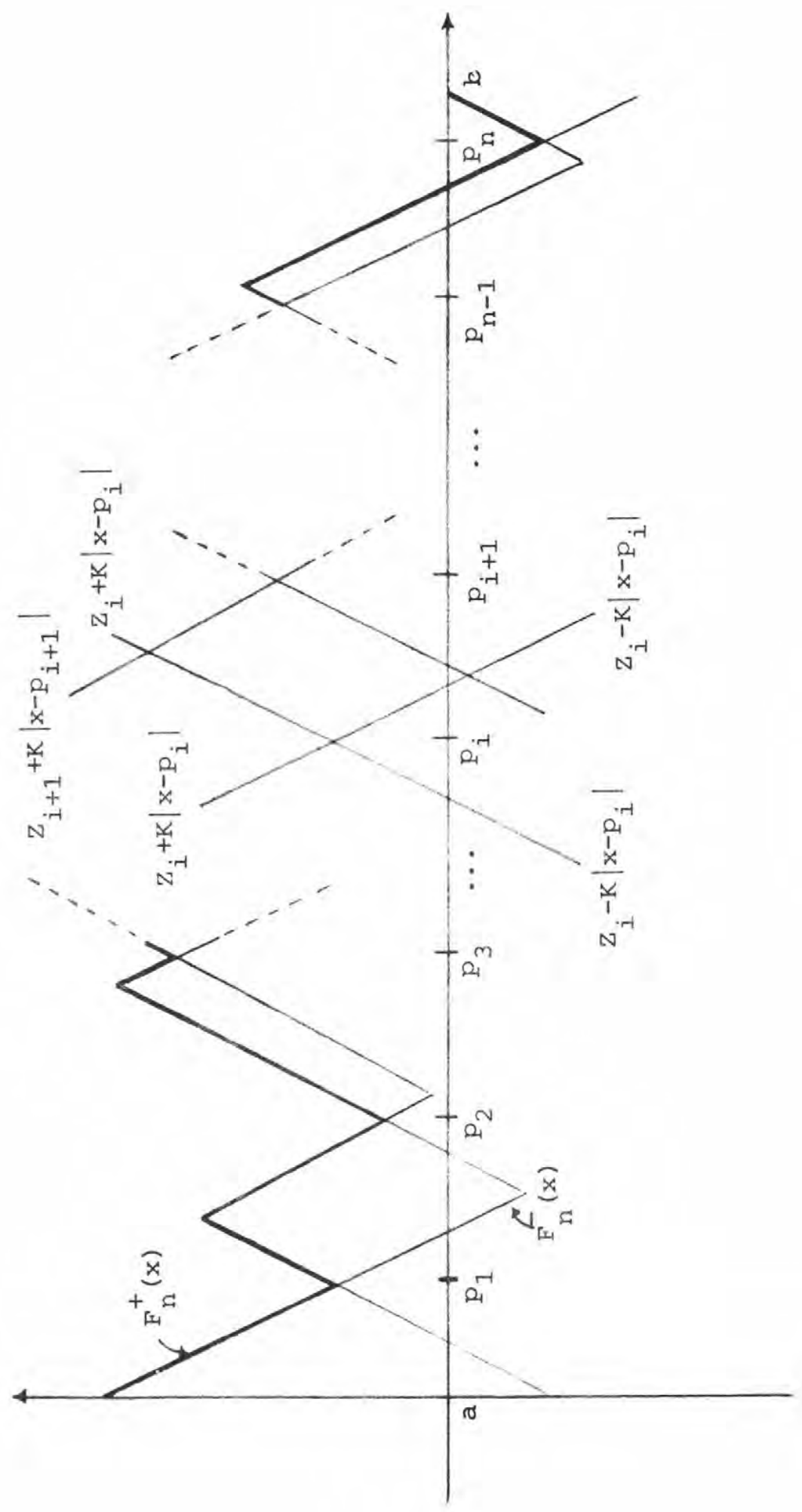

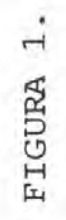




$$
\begin{aligned}
& F_{n, 0}^{-}(x)=z_{1}-k\left|x-p_{1}\right| \\
& F_{n, 0}^{+}(x)=z_{1}+k\left|x-p_{1}\right| \\
& F_{n, n}^{-}(x)=z_{n}-k\left|x-p_{n}\right| \\
& F_{n, n}^{+}(x)=z_{n}+k\left|x-p_{n}\right|
\end{aligned}
$$

(Ver Figura 2b).

1.2. Raíces y Valores Extremos de $\mathrm{F}_{n, i}^{-}$y $\mathrm{F}_{n, i}^{+}$.

Sean

$$
\begin{aligned}
& z_{i}^{* *}=\max _{x \in\left[p_{i}, p_{i+1}\right]} F_{n, i}^{+}(x) \\
& z_{i}^{*}=\min _{x \in\left[p_{i}, p_{i+1}\right]} F_{n, i}^{-}(x) \text {, para } i \in\{1, \ldots, n-1\}
\end{aligned}
$$

Es fácil probar que la solución de (11) está dada por

$$
\begin{aligned}
& z_{i}^{* *}=\frac{K}{2}\left(p_{i+1}-p_{i}\right)+\frac{z_{i}+z_{i+1}}{2} \\
& z_{i}^{*}=\frac{K}{2}\left(p_{i}-p_{i+1}\right)+\frac{z_{i}+z_{i+1}}{2}
\end{aligned}
$$

Lo importante de esto es que el único caso en que $\mathrm{F}_{n, i}^{-}$y/o $\mathrm{F}_{\mathrm{n}, i}^{+}$tienen raíces en $\left[\mathrm{p}_{i}, \mathrm{p}_{i+1}\right]$ es aquel en que $\mathrm{z}_{i}^{*} \leqslant 0 \quad \mathrm{y}^{\mathrm{n}} \mathrm{z}_{i}^{*} \geqslant 0$.

Se deduce sin mayor dificultad, que si $z_{i}^{*} \leqslant 0 y z_{i}^{* *} \geqslant 0$, enton ces el mayorante $\mathrm{F}_{n, i}^{+} \mathrm{Y}$ el minorante $\mathrm{F}_{n, i}^{-}$poseen en conjunto dos raíces en $\left[p_{i}, p_{i+1}\right]$, eventualmente confundidas, dadas por las relaciones:

$$
\begin{aligned}
& p_{1}^{i}=p_{i}+\frac{\left|z_{i}\right|}{K} \\
& p_{2}^{i}=p_{i+1}-\frac{\left|z_{i+1}\right|}{K}
\end{aligned}
$$




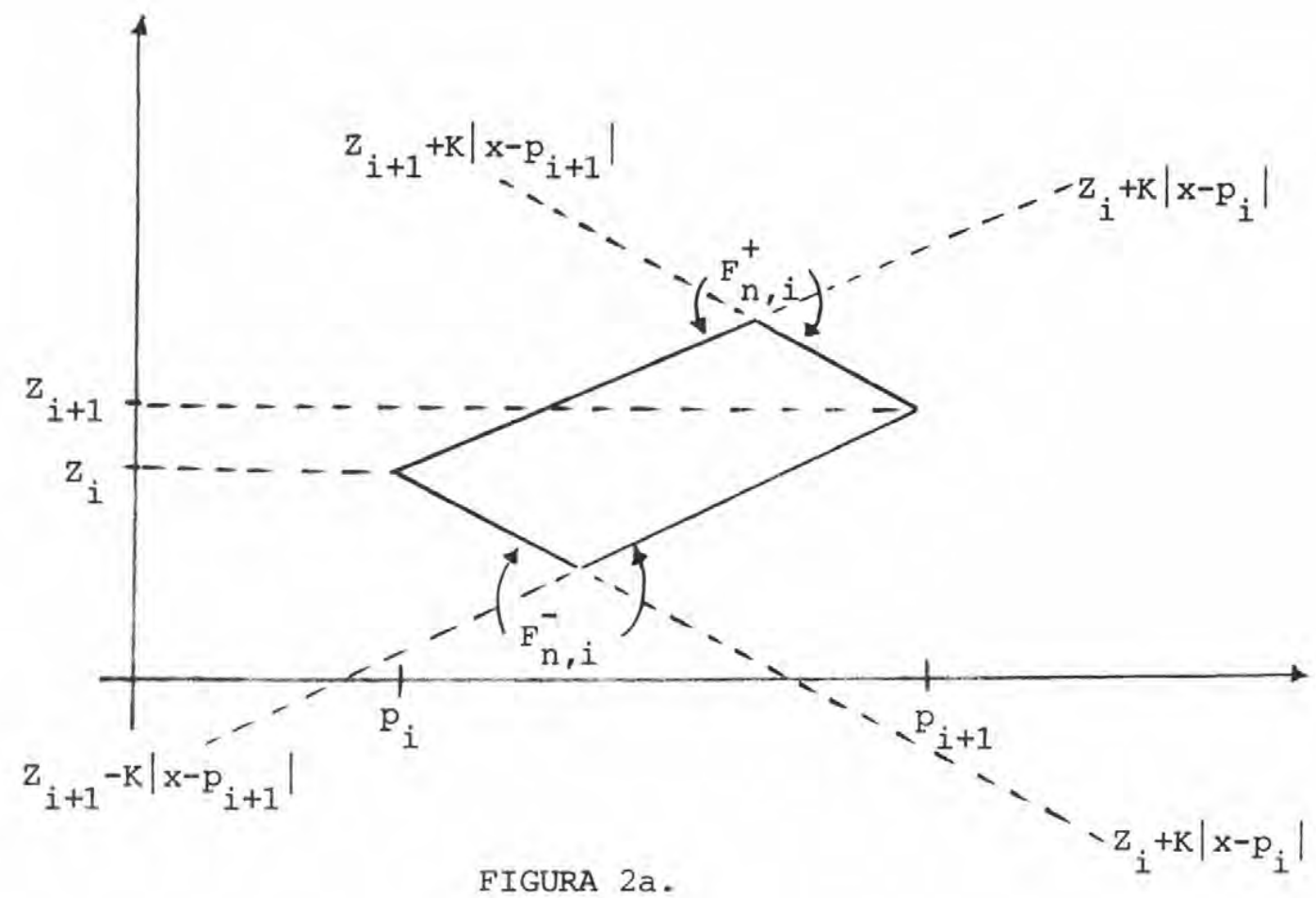

FIGURA 2a.
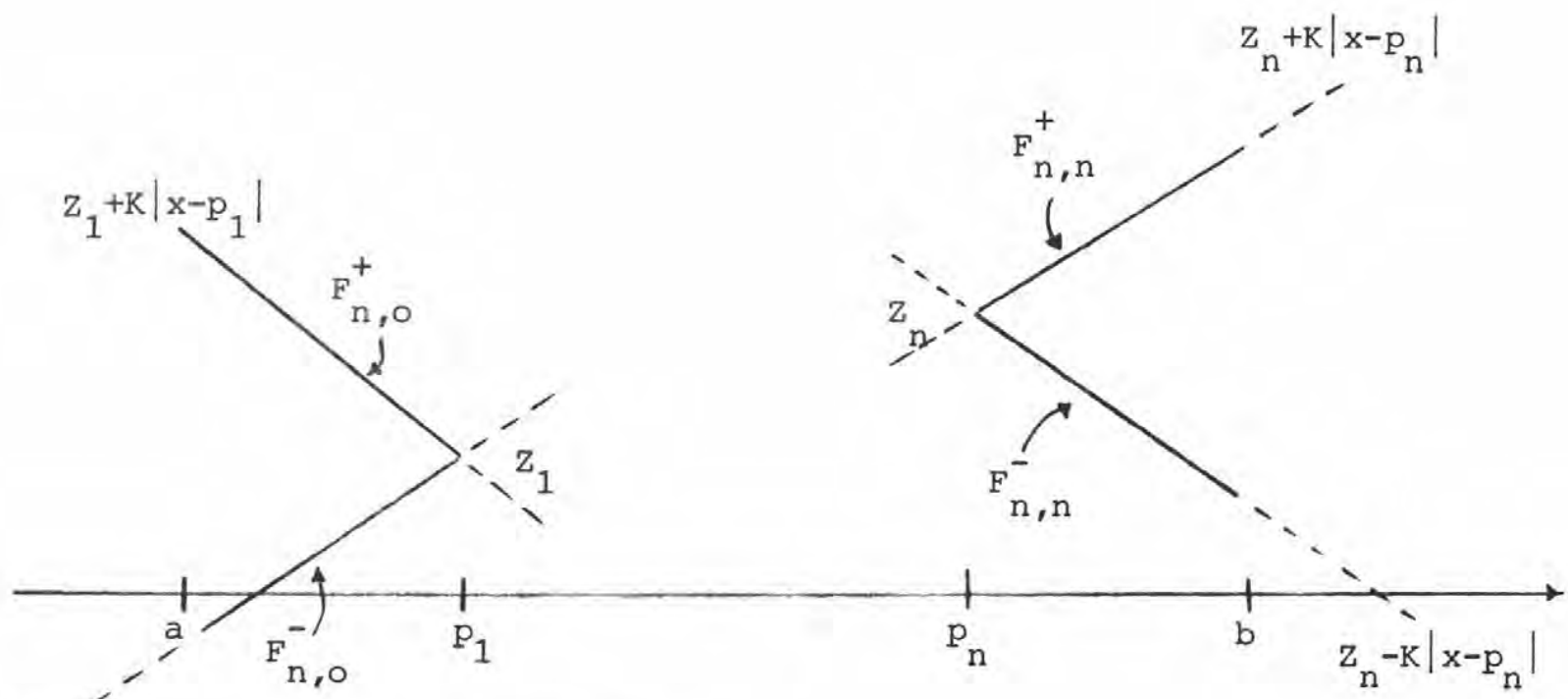

$z_{1}-\mathrm{k}\left|\mathrm{x}-\mathrm{p}_{1}\right|$

FIGURA 2b. 
En tal caso, debido a que toda función $g e \delta\left(f ; x_{1}, \ldots, x_{n}\right)$ tiene su gráfica en $\left[p_{i}, p_{i+1}\right]$ totalmente incluida en el paralelógramo $P_{i}$ definido por:

$$
P_{i}=\left\{(x, y) e \mathbb{R}^{2} / p_{i} \leqslant x \leqslant p_{i+1} ; F_{n, i}^{-}(x) \leqslant y \leqslant F_{n, i}^{+}(x)\right\} \text {, }
$$

se obtiene:

$$
R_{g}\left[p_{i}, p_{i+1}\right] \subseteq\left[p_{1}^{i}, p_{2}^{i}\right]
$$

(Ver Figura 3a)

De igual forma, si $\mathrm{F}_{n, i}^{-}$y $\mathrm{F}_{n, i}^{+}$no poseen raíces en $\left[p_{i}, p_{i+1}\right]$, es decir, si $z_{i}^{*}>0 \circ z_{i}^{* *}<0$, entonces:

$$
R_{g}\left[p_{i}, p_{i+1}\right]=\phi ; \forall g \in \delta\left(f ; x_{1}, \ldots, x_{n}\right)
$$

De acuerdo a lo anterior, la siguiente proposición es inmediata: Proposición 1. Sean $z_{i}^{* *}, z_{i}^{*}, p_{1}^{i}, p_{2}^{i}$ definidos por (12), (13) y (14) respectivamente. Entonces:

i) $z_{i}^{*} \leqslant 0$ y $z_{i}^{* *} \geqslant 0 \quad$ ssi $p_{1}^{i} \leqslant p_{2}^{i}$

ii) $\mathrm{F}_{\mathrm{n}, i}^{+}(\mathrm{x}) \geqslant 0, \mathrm{~F}_{\mathrm{n}, \mathrm{i}}^{-}(\mathrm{x}) \leqslant 0 ; \forall \mathrm{x} \in\left[\mathrm{p}_{1}^{i}, \mathrm{p}_{2}^{i}\right]$

Nota 1. Para los intervalos $\left[a, p_{1}\right]$ y $\left[p_{n}, b\right]$ se establece $p_{0}=a$, $\mathrm{p}_{n+1}=\mathrm{b}, \mathrm{y}$ se obtienen los mismos resultados de proposición 1 con:

$$
\begin{aligned}
& z_{\circ}^{* *}=\max _{x \in\left[p_{0}, p_{1}\right]} F_{n, o}^{+}(x)=z_{1}+k\left|a-p_{1}\right| \\
& z_{\circ}^{*}=\min _{x \in\left[p_{0}, p_{1}\right]} F_{n, o}^{-}(x)=z_{1}-k\left|a-p_{1}\right|
\end{aligned}
$$




$$
\begin{aligned}
& z_{n}^{* *}=\max _{x \in\left[p_{n}, p_{n+1]}\right.} F_{n, n}^{+}(x)=z_{n}+k\left|b-p_{n}\right| \\
& z_{n}^{*}=\min _{x \in\left[p_{n}, p_{n+1}\right]} F_{n, n}^{-}(x)=z_{n}-k\left|b-p_{n}\right| \\
& \mathrm{p}_{1}^{\circ}=\mathrm{a}, \mathrm{p}_{2}^{\circ}=\mathrm{p}_{1}-\frac{\left|\mathrm{z}_{1}\right|}{\mathrm{K}} \\
& p_{1}^{n}=p_{n}+\frac{\left|z_{n}\right|}{k}, p_{2}^{n}=b
\end{aligned}
$$

(Ver Figura 3b).

Es decir, 10 que se quiere resaltar finalmente es el hecho que la proposición 1 es válida para todo $i \in\{0,1, \ldots, n\}$.

De las relaciones (14), (15), (16), de la proposición 1 y de la Nota 1 se obtiene finalmente el criterio a usar para ver la existencia de raíces de $f$ en $\left[p_{i}, p_{i+1}\right]$.

Test: Para cada $i \in\{0,1, \ldots, n\}$ se tiene:

i) $\mathrm{Si} \mathrm{p}_{1}^{i}>\mathrm{p}_{2}^{i}$, entonces:

$R_{g}\left[p_{i}, p_{i+1}\right]=\phi ; \forall g$ e $\delta\left(f ; x_{1}, \ldots, x_{n}\right), y$ en particular

$R_{f}\left[p_{i}, p_{i+1}\right]=\phi$.

ii) $\operatorname{si} p_{1}^{i} \leqslant p_{2}^{i}$, entonces:

$R_{f}\left[p_{i}, p_{i+1}\right] \leq\left[p_{1}^{i}, p_{2}^{i}\right]$ y en este caso, el intervalo $\left[p_{1}^{i}, p_{2}^{i}\right]$

se constituye en la localización más pequeña para $R_{f}\left[P_{i}, P_{i+1}\right]$ dada 1a información disponible. 


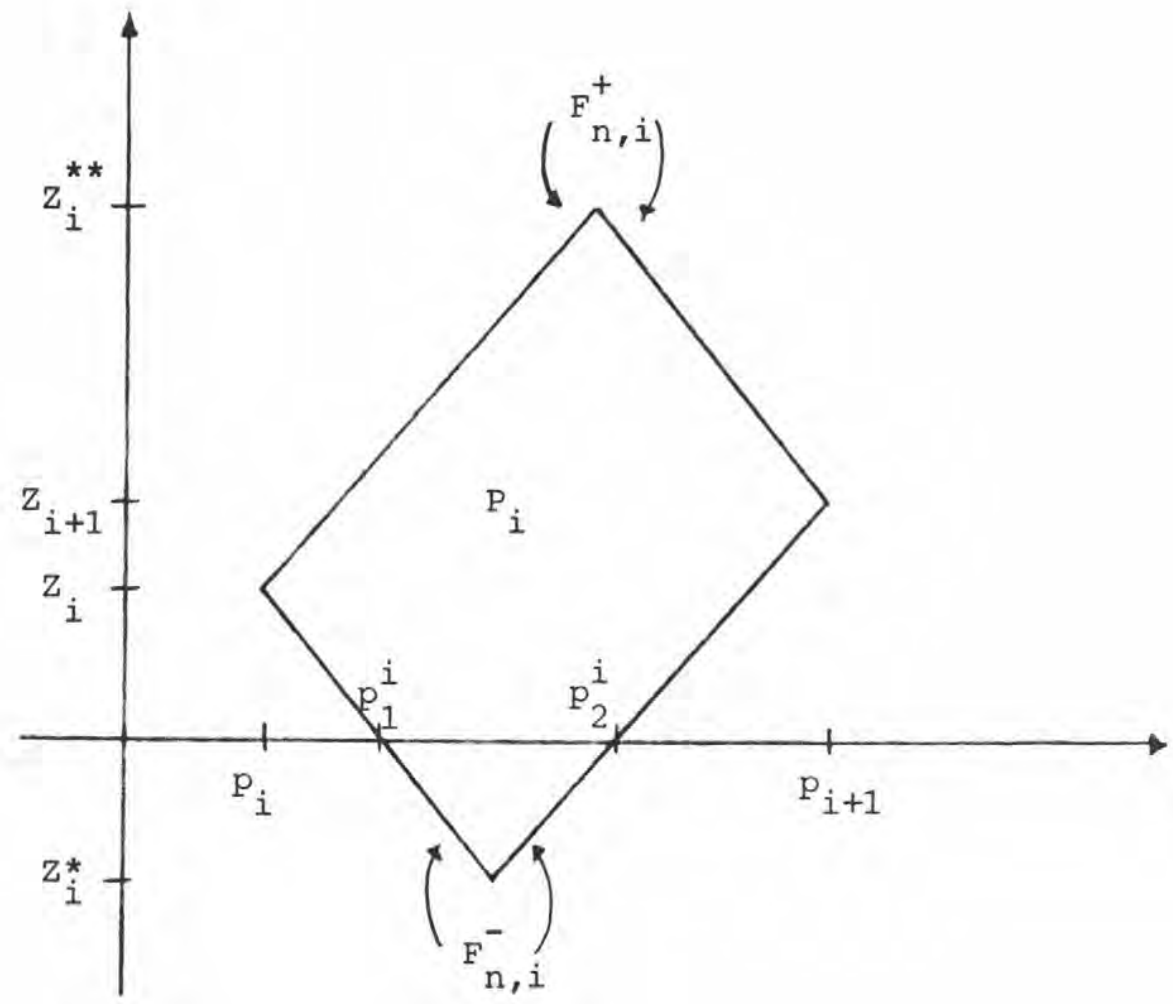

FIGURA $3 a$.

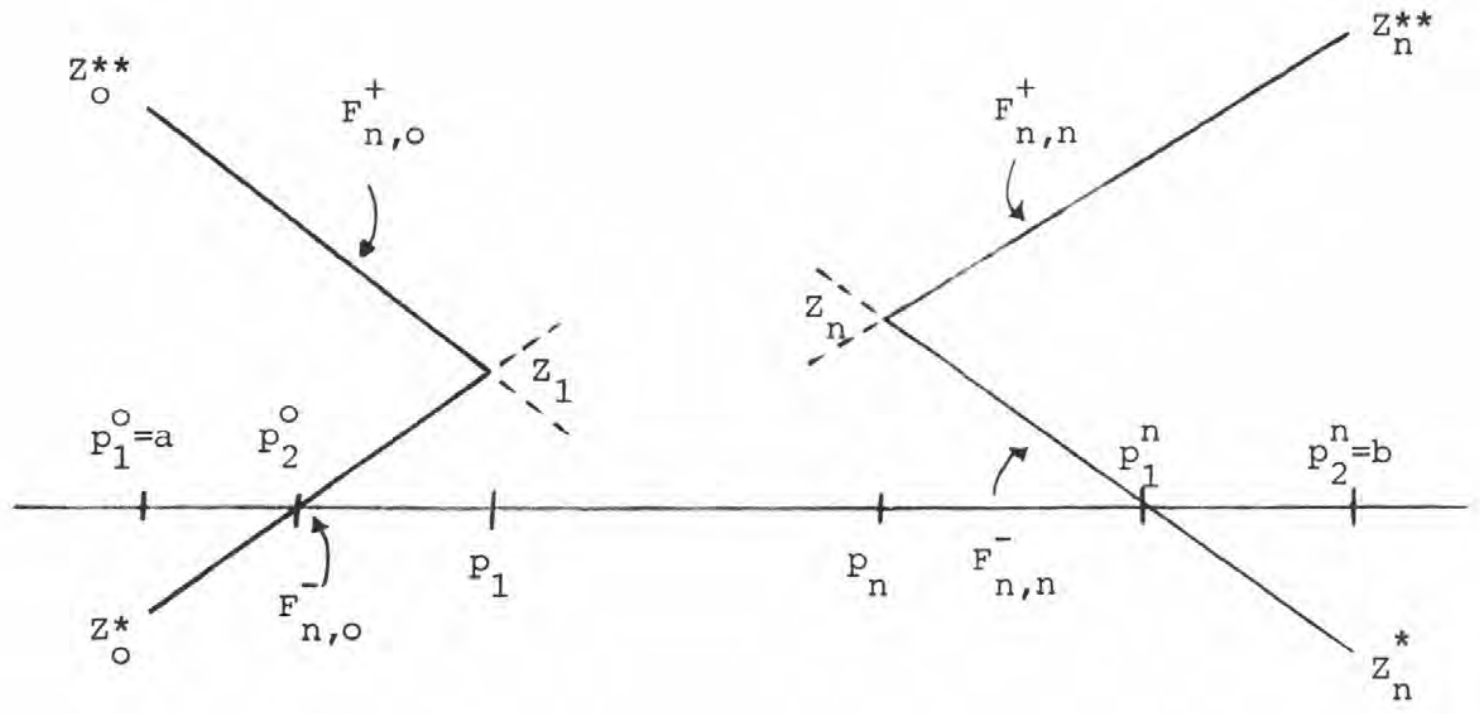

FIGURA 3b. 


\section{MODIFICACION DEL METODO SUKHAREV.}

Se define:

$$
L_{0}(K)=\left\{g \in \operatorname{Lip}[a, b]_{K} / R_{g}[a, b] \neq \phi\right\}
$$

Sea $f \in L_{\circ}(K)$ y supóngase que $f$ se ha evaluado en $n$ puntos $x_{1}, \ldots, x_{n}$ de $[a, b]$ tales que:

$$
\begin{aligned}
& \left\{p_{1}, \ldots, p_{n}\right\}=\left\{x_{1}, \ldots, x_{n}\right\} \\
& a \leqslant p_{1}<p_{2}<\ldots<p_{b} \leqslant b
\end{aligned}
$$

$\operatorname{con} z_{i}=f\left(p_{i}\right) ; i \in\{1, \ldots, n\}$

Se vió en [5] que con esta información SUKHAREV utiliza dos crí terios para desarrollar las estrategias óptima, mejor y los algoritmos de búsqueda. Se observó también que tales procedimientos no consideran los subintervalos, determinados por los puntos de evaluación y conducen a la obtención de sólo una raíz de la función. Aquí no se definirán criterios y por 10 tanto no se podrá hablar de estrategias óptimas. Sin embargo, se diseña un método convergente, el cual utiliza cada vez todos los intervalos de la forma $\left[a, p_{1}\right],\left[p_{i}, p_{i+1}\right]$ y $\left[p_{n}, b\right]$ para construir una sucesión de localizaciones de $R_{f}[a, b]$ decreciente en el senti do de inclusión.

Claramente se cumple que:

$$
R_{f}[a, b]=\bigcup_{i=0}^{n} R_{f}\left[p_{i}, p_{i+1}\right]
$$

Luego, el conjunto:

$$
L=\underset{i}{i} p_{1}^{i} \leqslant p_{2}^{i}\left[p_{1}^{i}, p_{2}^{i}\right]
$$

constituye una localización de $R_{f}[a, b]$. 


\subsection{Notación y Definiciones Previas.}

Se llamará iteración a aquel proceso en que, con la información disponible y en base a una o más evaluaciones de la función, se calcula una nueva localización de $\mathrm{R}_{\mathrm{f}}[\mathrm{a}, \mathrm{b}]$.

Supóngase que al cabo de la n-ésima iteración se han realizado $e(n)$ evaluaciones: $x_{1}, x_{2}, \ldots, n_{e(n)}, y$ se dispone de una localización $I_{n}$ del conjunto solución definida por la unión de $m_{n}$ intervalos cerrados $I_{n, j} ; j=\overline{1, m n}$; es decir:

$$
L_{n}=U_{j=1}^{m} I_{n, j}
$$

donde:

$$
I_{n, j}=\left[a_{n, j}, b_{n, j}\right] ; \forall j \in\left\{1, \ldots, m_{n}\right\}
$$

Sea además:

$$
\begin{aligned}
& T_{n}=\left\{I_{n, j} / j=\overline{1, m n}\right\} \\
& \lambda_{n}=\frac{\max }{j=1, m_{n}}\left(b_{n, j}-a_{n, j}\right)
\end{aligned}
$$

Siguiendo las notaciones dadas en 1.1., el mayorante y minorante de $f$, definidos en (7), se expresan como:

$$
\begin{aligned}
& F_{e(n)}^{+}(x)=\frac{\min }{j=1, e(n)}\left\{f\left(x_{j}\right)+k\left|x-x_{j}\right|\right\} \\
& F_{e(n)}^{-}(x)=\frac{\max }{j=1, e(n)}\left\{f\left(x_{j}\right)-K\left|x-x_{j}\right|\right\}
\end{aligned}
$$

Se definen también las siguientes funciones para cada localización $\mathrm{L}_{\mathrm{n}}$ :

$$
\mathrm{E}_{\mathrm{n}}^{+}:[\mathrm{a}, \mathrm{b}] \rightarrow \mathbb{R}
$$




$$
\begin{aligned}
E_{n}^{+}(x) & =\left\{\begin{array}{l}
f(x) ; x \notin L_{n} \\
F_{e(n)}^{+}(x) ; x \in I_{n}
\end{array}\right. \\
E_{n}^{-} ;[a, b] \rightarrow \mathbb{R} & E_{n}^{-}(x)=\left\{\begin{array}{l}
f(x) ; x \notin L_{n} \\
F_{e(n)}^{-}(x) ; x \in L_{n}
\end{array}\right.
\end{aligned}
$$

De acuerdo a los resultados dados en 1.1. se obtiene claramente

$$
E_{n}^{-}(x) \leqslant f(x) \leqslant E_{n}^{+}(x) ; \forall x \in[a, b] .
$$

El método que se detallará a continuación, sigue la idea del pro cedimiento de base y de los algoritmos 1 y 2 desarrollados por BASSO [2]. Estas técnicas, a su vez, provienen de los trabajos de localización de raíces de polinomios en el plano complejo, los cuales consisten en dividir en cada paso una región de $\mathbf{C}$ que contiene todas las raíces y testear cada una de estas partes para detectar los conjuntos susceptibles de con tener a una.

\subsection{Algoritmo.}

Dado $r \in \mathbb{N}$ se construye aquí una sucesión de localizaciones $\left\{L_{n}\right\}$ de $R_{f}[a, b]$ del tipo $L$ dado en (18) mediante el procedimiento básico siguiente: en cada elemento $I_{n, j}$ de $T_{n}$ se determinan $r+1$ subintervalos de igual longitud introduciendo $r$ puntos de evaluación en $I_{n, j}$ y se ana liza en cada uno de ellos, mediante el test dado en 1.2 la existencia de raíces para $\mathrm{f}$.

Los pasos del algoritmo son:

INICIO: $L_{1}=[a, b] ; m_{1}=1 ; I_{1,1}=[a, b] ; \lambda_{1}=b-a ; n=1$ 
PASO 1: $T_{n+1}=L_{n+1}=\phi ; m_{n+1}=0 ; \lambda_{n+1}=0$

PASO 2: Para cada intervalo $I_{n, j}=\left[a_{n, j}, b_{n, j}\right] \in T_{n}$ hacer 10 siguiente:

2.1.) Calcular $h_{n, j}=\frac{b_{n, j}-a_{n, j}}{r+1}$

2.2.) Calcular $x_{n, j}^{p}=a_{n, j}+p h_{n, j} ; p=\overline{1, r}$

2.3.) Testear los intervalos $\left[a_{n, j^{\prime}}, x_{n, j}^{1}\right] y\left[x_{n, j}^{r}, b_{n, j}\right]$

$$
\begin{aligned}
& \text { 2.3.1.) Hacer } x=x_{n, j}^{1}-\frac{\left|f\left(x_{n, j}^{1}\right)\right|}{k} \\
& \text { Si } a_{n, j}>x \text {, entonces } R_{f}\left[a_{n, j}, x_{n, j}^{1}\right]=\phi \text {. } \\
& \text { En caso contrario } R_{f}\left[a_{n, j}, x_{n, j}^{1} \subseteq\left[a_{n, j}, x\right]\right. \\
& y \text { se hace: } \\
& m_{n+1}=m_{n+1}+1 ; T_{n+1}=T_{n+1} U\left\{\left[a_{n, j}, x\right]\right\} \\
& L_{n+1}=L_{n+1} U\left[a_{n, j}, x\right] ; \lambda_{n+1}=\max \left\{\lambda_{n+1} ; x-a_{n, j}\right\} \\
& \text { 2.3.2.) Hacer } x=x_{n, j}^{r}+\frac{\left|f\left(x_{n, j}^{r}\right)\right|}{k}
\end{aligned}
$$$$
\text { Si } x>b_{n, j} \text {, entonces } R_{f}\left[x_{n, j}^{r}, b_{n, j}\right]=\phi
$$$$
\text { En caso contrario } R_{f}\left[x_{n, j}^{x}, b_{n, j}\right] \subseteq\left[x, b_{n, j}\right] \text { y se ha- }
$$

ce:

$$
\begin{aligned}
& m_{n+1}=m_{n+1}+1 ; T_{n+1}=T_{n+1} \cup\left\{\left[x, b_{n, j}\right]\right\} \\
& L_{n+1}=I_{n+1} U\left[x, b_{n, j}\right] ; \lambda_{n+1}=\max \left\{\lambda_{n+1} ; b_{n, j}-x\right\}
\end{aligned}
$$


2.4.) Testear los intervalos $\left[x_{n, j^{\prime}}^{p}, x_{n, j}^{p+1}\right] ; p=\overline{1, r-1}$

Hacer $x^{-}=x_{n, j}^{p}+\frac{f\left(x_{n, j}^{p}\right)}{k} ; x^{+}=x_{n, j}^{p+1}-\frac{f\left(x_{n, j}^{p+1}\right.}{k}$

Si $x^{-}>x^{+}$, entonces $R_{f}\left[x_{n, j}^{p}, x_{n, j}^{p+1}\right]=\phi$. En caso contrario

$R_{f}\left[x_{n, j^{p}}^{p}, x_{n, j}^{p+1}\right] \subseteq\left[x^{-}, x^{+}\right]$y se hace:

$m_{n+1}=m_{n+1}+1 ; T_{n+1}=T_{n+1} U\left\{\left[x^{-}, x^{+}\right]\right\}$

$L_{n+1}=L_{n+1} U\left[x^{-}, x^{+}\right] ; \lambda_{n+1}=\max \left\{\lambda_{n+1} ; x^{+}-x^{-}\right\}$

PASO 3: Realizar test de detención (por ejemplo $\lambda_{n}<\delta$ ). Si es positi vo ir a paso 4 , si no lo es, hacer $n=n_{-}$e ir a paso 1 .

PASO 4: Detener el proceso.

Nota 2. El algoritmo descrito merece los siguientes comentarios:

i) El procedimiento corxesponde a un método de tipo Block-secuencial (realiza varias evaluaciones simultáneas en forma secuencial) aná logo a los desarrollados para localización del máximo global (Véa se BASSO [1] y en los que se realiza al menos una evaluación en cada uno de los intervalos disjuntos que componen la localización.

ii) El caso $r=1$ corresponde a la técnica del punto medio aplicada a cada uno de los intervalos $\left[a_{n, j}, b_{n, j}\right] \in T_{n}$.

iii) Cuando en el paso 2 se testea un intervalo $\left[x_{n, j}^{p}, x_{n, j}^{p+1}\right]$ y ocurre que

$$
x_{n, j}^{p}+\frac{\left|f\left(x_{n, j}^{p}\right)\right|}{k}>x_{n, j}^{p+1} \text {, entonces obviamente }
$$


$R_{f}\left[x_{n, j}^{p}, x_{n, j}^{p+1}\right]=\phi$. Sin embargo, lo que interesa notar aquí es que $\mathrm{si}$ existe $q>p+1$ tal que:

$$
x_{n, j}^{p}+\frac{\left|f\left(x_{n, j}^{p}\right)\right|}{k}>x_{n, j}^{q}
$$

entonces $R_{f}\left[x_{n, j}^{p}, x_{n, j}^{q}\right]=\phi y$ se hace innecesario evaluar $f$ en los puntos $x_{n, j}^{s} ; p<s<q$. Es por esto que en vez de calcular de an temano la función en los $r$ puntos $x_{n, j}^{p} ; p=\overline{1, r}$, conviene ir evaluando a medida que se van testeando los subintervalos, y a la vez se debe ir analizando la posibilidad (28).

iv) Del paso 2 se observa que cada elemento de $T_{n+1}$ es un subintervalo de algún elemento de $T_{n}$. Además, si el intervalo $I_{n+1, p} \in T_{n+1}$

$\forall p \in\left\{1, \ldots, m_{n+1}\right\}$, proviene del elemento $I_{n, q} \in T_{n} ; \forall q \in\{1$, $\left.\ldots, m_{n}\right\}$, entonces es claro que:

$$
b_{n+1, p}-a_{n+1, p} \leqslant \frac{b_{n, q}-a_{n, q}}{x+1}
$$

De aquí se concluye el importante resultado:

$$
\max \left(b_{n+1, j}-a_{n+1, j}\right) \leqslant \frac{1}{r+1} \quad \max \left(B_{n, j}-a_{n, j}\right)
$$

$$
j \in\left\{1, \ldots, m_{n+1}\right\} \quad j \in\left\{1, \ldots, m_{n}\right\}
$$

es decir:

$$
\lambda_{\mathrm{n}+1} \leqslant \frac{\lambda_{\mathrm{n}}}{r+1} ; \quad \forall \mathrm{n} \in \mathbf{N}
$$

Se tiene la siguiente proposición:

Proposición 2. Bajo este algoritmo:

$$
\lim _{n \rightarrow \infty} \lambda_{n}=0
$$


Demostración: Aplicando sucesivamente la relación (29) resulta:

$\lambda_{n} \leqslant \frac{\lambda_{n-1}}{r+1} \leqslant \frac{\lambda_{n-2}}{(r+1)^{2}} \leqslant \cdots \leqslant \frac{\lambda_{1}}{(r+1)^{n-1}}=\frac{(b-a)}{(r+1)^{n-1}}$

como $r+1 \geqslant 2$, se obtiene: $\lim _{n \rightarrow \infty} \frac{(b-a)}{(r+1)^{n-1}}=0$ y por 10 tanto:

$\lim _{n \rightarrow \infty} \lambda_{n}=0$

otro resultado de interés es aquel que se establece a continuación:

Proposición 3. El mayorante $\mathrm{E}_{\mathrm{n}}^{+} \mathrm{y}$ el minorante $\mathrm{E}_{\mathrm{n}}^{-}$dados por (25) y (26) convergen puntualmente a la función $f$ en $[a, b]$. Esto es:

$$
\lim _{n \rightarrow \infty} E_{n}^{-}(x)=\lim _{n \rightarrow \infty} E_{n}^{+}(x)=f(x) ; \forall x \in[a, b]
$$

Demostración:

Sea $x \in[a, b]$. Si $x \notin L_{m^{\prime}}$ para algún $m \in \mathbb{N}$, entonces $x \notin L_{n}, \forall n \geqslant m$. Luego:

$$
\begin{aligned}
& E_{n}^{+}(x)=E_{n}^{-}(x)=f(x), \forall n \geqslant m, y \text { obviamente: } \\
& \lim _{n \rightarrow \infty} E_{n}^{+}(x)=\lim _{n \rightarrow \infty} E_{n}^{-}(x)=f(x) \\
& \text { Si } x \in L_{n}, \forall n \in \mathbb{N} \text {, entonces se denota por } \\
& I_{n, n(x)}=\left[a_{n, n(x)}, b_{n, n(x)}\right] \text { el elemento de } T_{n} \text { al cual pertene }
\end{aligned}
$$

ce $z$. Para efectos de simplicidad se escribe:

$$
I_{n, n(x)}=I_{n}=\left[a_{n}, b_{n}\right]
$$




$$
\text { Sean } x_{n}^{\circ}=a_{n}, x_{n}^{r+1}=b_{n} y x_{n}^{p}=a_{n}+p \frac{\left(b_{n}-a_{n}\right)}{r+1} ; p=\overline{1, r},
$$

los $r$ puntos de evaluación considerados en el paso 2.2 del algoritmo para el intervalo $I_{n}$.

Sea $k \in\{0,1, \ldots, r\}$ tal que $x \in\left[x_{n}^{k}, x_{n}^{k+1}\right]$

Se define:

$$
\bar{x}_{n}=\left\{\begin{array}{l}
x_{n}^{1} ; \text { si } k=0 \\
x_{n}^{r} ; \text { si } k=r \\
x_{n}^{k} ; \text { si } k \in\{1, \ldots, x-1\}
\end{array}\right.
$$

entonces $\bar{x}_{n} \in\left\{x_{n}^{1}, \ldots, x_{n}^{Y}\right\} ;$ es decir $\bar{x}_{n}$ es un punto de evaluación de $f$ para iteración $n+1$, 10 que es equivalente a escribir:

$$
\bar{x}_{n} \in\left\{x_{1}, x_{2}, \ldots, x_{e(n+1)}\right\}
$$

Además:

$$
\left|\vec{x}_{n}-x\right| \leqslant\left|x_{n}^{k+1}-x_{n}^{k}\right|=\frac{b_{n}-a_{n}}{r+1} \leqslant \frac{\lambda_{n}}{r+1}
$$

$y$ puesto que $\lim _{n \rightarrow \infty} \lambda_{n}=0$ (Por proposición 2), se deduce:

$$
\lim _{n \rightarrow \infty} \bar{x}_{n}=x
$$

Por otro lado, como $x \in L_{n+1}$ se tiene $E_{n+1}^{+}(x)=F_{e(n+1)}^{+}(x)$ es decir:

$$
\begin{aligned}
& E_{n+1}^{+}(x)=\frac{\min }{j=1, e(n+1)}\left\{f\left(x_{j}\right)+k\left|x-x_{j}\right|\right\} \\
& \text { De (30) y (32) se obtiene: }
\end{aligned}
$$

$$
E_{n+1}^{+}(x) \leqslant f\left(\bar{x}_{n}\right)+K\left|x-\bar{x}_{n}\right|
$$


Se tiene entonces:

$$
f(x) \leqslant E_{n+1}^{+}(x) \leqslant f\left(\bar{x}_{n}\right)+K\left|x-\bar{x}_{n}\right|
$$

Tomando límite cuando $n \rightarrow \infty$ y recordando la continuidad de $f$ y el hecho que

$$
\begin{aligned}
\bar{x}_{n} \stackrel{n \rightarrow \infty}{\longrightarrow} x, \text { se concluye: } \\
f(x) \leqslant \lim _{n \rightarrow \infty} E_{n+1}^{+}(x) \leqslant f(x) \text {, o bien } \\
\\
\lim _{n \rightarrow \infty} E_{n}^{+}(x)=f(x)
\end{aligned}
$$

De la misma forma:

$f(x) \geqslant E_{n+1}^{-}(x)=\frac{\max }{j=1, e(n+1)}\left\{f\left(x_{j}\right)-k\left|x-x_{j}\right|\right\} \geqslant f\left(\bar{x}_{n}\right)-K\left|x-\bar{x}_{n}\right|$

y anälogamente se obtiene:

$$
\lim _{n \rightarrow \infty} E_{n}^{-}(x)=f(x)
$$

Nota 3. Es importante notar en la demostración de la proposición 3 , concretamente en las desigualdades (33) y (34), que la rapidez con que el mayorante y el minorante convergen a $f$ depende fuertemente de la constante de Lipschitz K: mientras más pequeño sea este valor, más rápida será la convergencia de $\mathrm{E}_{n}^{-}$y $\mathrm{E}_{n}^{+}$a la función $\mathrm{f}$. Además, de la de sigualdad $\left|\bar{x}_{n}-x\right| \leqslant \frac{\lambda_{n}}{r+1}$ se observa que $\bar{x}_{n}$ se aproxima más rápido a $x$ si $r$ es grande, lo cual, sin embargo, produce el inconveniente de aumentar el número de evaluaciones de la función $\mathrm{f}$. 
Se demuestra finalmente que la sucesión de localizaciones determinadas por el algoritmo converge al conjunto solución $R_{f}[a, b]$.

Proposición 4. Se tiene:

$$
\mathrm{n}_{n=1}^{\infty} L_{n}=R_{f}[a, b]
$$

Demostración.

Es claro que por construcción de la sucesión $\left\{\mathrm{L}_{\mathrm{n}}\right\}$ resulta:

$$
\begin{aligned}
& R_{f}[a, b] \subseteq L_{n}, \forall n \in \mathbb{N} \text {. Luego: } \\
& R_{f}[a, b] \subseteq n_{n=1}^{\infty} L_{n}
\end{aligned}
$$

$\infty$

Sea $x \in \bigcap_{n=1} I_{n}$. Entonces $x \in L_{n}, \forall n \in \mathbb{N}$.

Sea $I_{n, n(x)}$ el elemento de $T_{n}$ al cual pertenece $x$. De acuerdo al algoritmo, este intervalo es de la forma $\left[p_{1}^{i}, p_{2}^{i}\right]$ descrita en 1.2 ; luego, por proposición $1 \mathrm{ii)}$ se deduce $\mathrm{F}_{\mathrm{e}(\mathrm{n})}^{+}(\mathrm{x}) \geqslant 0 \mathrm{y} \mathrm{F}_{\mathrm{e}(\mathrm{n})}^{-}(\mathrm{x}) \leqslant 0$.

Ahora de (25) y (26) se tiene que $E_{n}^{+} \mathrm{y} \mathrm{F}_{\mathrm{e}(\mathrm{n})}^{+}$(resp. $\mathrm{E}_{\mathrm{n}}^{-} \mathrm{y} \mathrm{F}_{\mathrm{e}(\mathrm{n})}^{-}$) coinciden sobre $L_{n}$. Así se obtiene:

$$
\begin{aligned}
& E_{n}^{+}(x) \geqslant 0, \forall n \in \mathbb{N} \\
& E_{n}^{-}(x) \leqslant 0, \forall n \in \mathbb{N}
\end{aligned}
$$

Tomando límite en (36) y (37) cuando $n \rightarrow \infty$, y usando proposición 3 , se concluye $f(x)=0$.

Esto indica que $x \in R_{f}[a, b]$ y por 10 tanto

$$
\bigcap_{n=1}^{\infty} L_{n} \subseteq R_{f}[a, b]
$$

De este modo, (35) y (38) completan la demostración. 


\section{ENSAYOS NUMERICOS.}

En esta sección se muestran algunos ejemplos de problemas de 10 calización de reíces sobre un intervalo en los que se usa el método modi ficado de SUKHAREV. Se hace notax que la constante de lipschitz se calcula sobr el intervalo total una sola vez y que ella no es recalculada para los subintervalos posteriores.

A medida que transucrran los ejemplos se observará que el nứnero de intervalos que componen la localización es, en general, mayor al número de raíces de la función. Esto se debe a que cuando $\lambda_{\mathrm{n}} \rightarrow 0, \mathrm{y}$ pro ducto de las sucesivas divisiones de los subintervalos, el test de exclu sión no puede eliminar todos los intervalos "parásitos" (que no contienen raíces) y ellos se siguen conservando alrededor de cada raíz. Por lo tanto, en cada iteración, para una raíz determinada habrá cierta cantidad de intervalos asociados a ella, de los cuales uno sólo la contiene.

A continuación se define la notación a emplear. Al cabo de la n-ésima iteración se escribe:

IT $=n$

$\mathrm{NEF}=$ número total de evaluaciones de $\mathrm{f}$.

NINT = número de intervalos que componen la localización $=\mathrm{m}_{\mathrm{n}}$.

LONT = longitud total de la localización

$$
=\sum_{j=1}^{m}\left(b_{n, j}-a_{n, j}\right)
$$

LONMAX $=$ longitud máxima en la localización $=\lambda_{\mathrm{n}}$.

$[a(j), b(j)]=$ intervalo de $T_{n}$ que contiene a la j-ésima raíz.

$n(j)=$ número de intervalos asociados a la j-ésima raíz. 


\subsection{Ejemplo 1 .}

Se considera $\mathrm{f}: \mathbb{R} \rightarrow \mathbb{R}$ definida por:

$f(x)=(1.4-3 x)$ sen $(18 x), y$ se quiere determinar $R_{f}[0,1.2]$. Esta función tiene 8 raíces en [0, 1.2]. El ejemplo ha sido tomado de BASSO [2] : (III. 2, ejemplo 1).

Se trabaja con una constante de Lipschitz $\mathrm{K}=42.6$ la cual se obtiene por mayoración de $\left|f^{\prime}(x)\right|$ en $[0,1.2]$.

La tabla 1 muestra el comportamiento del método para $r=1$.

Tabla $1 \quad(r=1)$

\begin{tabular}{|c|c|c|c|c|}
\hline IT & NEF & NINT & LONT & LONMAX \\
\hline 1 & 1 & 2 & $0.118 D+01$ & $0.591 D+00$ \\
\hline 2 & 3 & 4 & $0.113 \mathrm{D}+01$ & $0.285 D+00$ \\
\hline 3 & 7 & 8 & $0.105 \mathrm{D}+01$ & $0.141 D+00$ \\
\hline 4 & 15 & 16 & $0.755 \mathrm{D}+00$ & $0.705 \mathrm{D}-01$ \\
\hline 5 & 31 & 22 & $0.481 D+00$ & $0.344 \mathrm{D}-01$ \\
\hline 6 & 53 & 32 & $0.300 D+00$ & $0.172 \mathrm{D}-01$ \\
\hline 7 & 85 & 42 & $0.173 \mathrm{D}+00$ & $0.814 \mathrm{D}-02$ \\
\hline 8 & 127 & 52 & $0.992 \mathrm{D}-01$ & $0.384 \mathrm{D}-02$ \\
\hline 9 & 179 & 64 & $0.527 \mathrm{D}-01$ & $0.181 \mathrm{D}-02$ \\
\hline 10 & 243 & 56 & $0.210 D-01$ & $0.860 D-03$ \\
\hline 11 & 299 & 54 & $0.975 \mathrm{D}-02$ & $0.411 D-03$ \\
\hline 12 & 353 & 52 & $0.474 \mathrm{D}-02$ & $0.199 \mathrm{D}-03$ \\
\hline 13 & 405 & 54 & $0.209 \mathrm{D}-02$ & $0.993 D-04$ \\
\hline 14 & 459 & 52 & $0.969 D-03$ & $0.472 \mathrm{D}-04$ \\
\hline 15 & 511 & 54 & $0.493 \mathrm{D}-03$ & $0.228 D-04$ \\
\hline 16 & 565 & 52 & $0.234 \mathrm{D}-03$ & $0.113 \mathrm{D}-04$ \\
\hline 17 & 617 & 56 & $0.112 \mathrm{D}-03$ & $0.540 D-05$ \\
\hline 18 & 673 & 54 & $0.532 \mathrm{D}-04$ & $0.264 D-05$ \\
\hline
\end{tabular}


Se observa ahí que el crecimiento del número de intervalos comienza desde $I T=3$, pero a partir de $I T=10$, el valor NINT permanece más o menos fijo oscilando alrededor de NINT $=54$. Esto quiere decir que cuando LONT es menor que $10^{-1}$ el test de exclusión no elimina todos los intervalos parásitos y cada vez que se divide alguno de ellos, al menos uno de sus dos subintervalos se mantiene en la localización. Por otra parte, el decrecimiento de LONT es lento, debido en gran parte a que $\mathrm{K}$ es relativamente grande.

La tabla 2 muestra la aproximación a $R_{f}[0,1.2]$ al cabo de IT $=18$. Cada raíz puede obtenerse con al menos cinco cifras decimales correctas. Puede apreciarse también que la 4a. y 5a. raíz son las más afectadas con el crecimiento del número de intervalos. En general, cier tas soluciones se localizan, para una misma iteración, con más precisión que otras y/o con más intervalos adyacentes. Esto se debe, por una parte, a la posición de los ceros con respecto a los puntos donde se evalúa la función y también a la forma de la curva alrededor de la raíz.

\section{Tabla $2 \quad(r=1, \quad I T=18)$}

\begin{tabular}{|cccc|}
\hline$j$ & $a(j)$ & $b(j)$ & $n(j)$ \\
\hline 1 & $\underline{0.000000000000000000 D+00}$ & $0.952913738281166377 D-11$ & 2 \\
2 & $\underline{0.174532839217647076 D+00}$ & $\underline{0.174532995630700635 D+00}$ & 4 \\
3 & $\underline{0.349065139692671658 D+00}$ & $\underline{0.349066597624719553 D+00}$ & 8 \\
4 & $\underline{0.466665701668943596 D+00}$ & $\underline{0.466668340274675912 D+00}$ & 18 \\
5 & $\underline{0.523596447873232344 D+00}$ & $\underline{0.523598928618734424 D+00}$ & 14 \\
7 & $\underline{0.698131336946914045 D+00}$ & $\underline{0.698131714070141257 D+00}$ & 4 \\
8 & $\underline{0.872664622946473079 D+00}$ & $\underline{0.872664665102541892 D+00}$ & 2 \\
\hline
\end{tabular}




\subsection{Ejemplo 2.}

Se considera $f: \mathbb{R} \rightarrow \mathbb{R}$ definida por

$f(x)=\left(e^{x}-5 x^{2}\right)$ sen $(15 x), y$ se quiere determinar $R_{f}[-1,1]$. Esta fun ción tiene 11 raíces en $[-1,1]$.

Este ejemplo constituye un caso en el que los métodos para funciones Lipschitz continuas resultan prácticamente inutilizables. A modo de confirmación para esto, se obtiene por mayoración de $\left|f^{\prime}(x)\right|$ en $[-1,1]$ una constante de Lipschitz $\mathrm{K}=80$.

La tabla 3 muestra un resumen del comportamiento del método (para $r=1$ ) con respecto a las cantidades IT, NEF y NINT.

$$
\text { Tabla } 3 \quad(r=1)
$$

\begin{tabular}{|rrr|}
\hline IT & NEF & NINT \\
\hline 3 & 7 & 8 \\
6 & 61 & 52 \\
7 & 113 & 76 \\
8 & 189 & 96 \\
9 & 285 & 100 \\
10 & 385 & 134 \\
11 & 519 & 182 \\
12 & 701 & 192 \\
13 & 893 & 248 \\
14 & 1141 & 210 \\
15 & 1351 & 216 \\
16 & 1567 & 208 \\
\hline
\end{tabular}


Se aprecia aquí que se produce una alta cantidad de intervalos de localización en comparación con el número de raíces de f. Es decir, es mayoritaria la presencia de intervalos parásitos después de cada ite ración. Sin embargo, pese a que el valor NINT comienza a crecer a partir de IT $=3$, él se estabiliza desde IT $=13$ en adelante, alrededor de, aproximadamente, NINT $=210$. Esta característica hace, a su vez, que el número de evaluaciones de f aumente rápidamente.

\subsection{Ejemplo 3 .}

Con el objeto de estudiar el comportamiento del método ante un gran número de raíces se considera aquí la función $f \mathbb{R} \rightarrow \mathbb{R}$ definida por: $f(x)=\sum_{k=1}^{5} k \operatorname{sen}((k+1) x+k)$, la cual tiene 38 raíces en el intervalo $[-10,10]$. Este ejemplo ha sido tomado de BASSO [2]: (III. 2, ejemplo 5)

Se trabaja con una constante de tipschitz $K=70$, la cual se ob tiene por mayorazión de $\left|f^{\prime}(x)\right|$ en $[-10,10]$.

La tabla 4 muestra los resultados obtenidos con el método $($ para $r=1)$ 
Tabla $4 \quad(r=1)$

\begin{tabular}{|rrrrr|}
\hline IT & NEF & NINT & LONT & LONMAX \\
\hline 2 & 3 & 4 & $0.195 \mathrm{D}+02$ & $0.494 \mathrm{D}+01$ \\
4 & 15 & 16 & $0.182 \mathrm{D}+02$ & $0.119 \mathrm{D}+01$ \\
6 & 63 & 64 & $0.131 \mathrm{D}+02$ & $0.270 \mathrm{D}+00$ \\
8 & 239 & 154 & $0.404 \mathrm{D}+01$ & $0.541 \mathrm{D}-01$ \\
10 & 531 & 136 & $0.630 \mathrm{D}+00$ & $0.107 \mathrm{D}-01$ \\
11 & 667 & 132 & $0.277 \mathrm{D}+00$ & $0.471 \mathrm{D}-02$ \\
12 & 799 & 132 & $0.113 \mathrm{D}+00$ & $0.228 \mathrm{D}-02$ \\
13 & 931 & 132 & $0.497 \mathrm{D}-01$ & $0.914 \mathrm{D}-03$ \\
14 & 1063 & 126 & $0.201 \mathrm{D}-01$ & $0.395 \mathrm{D}-03$ \\
15 & 1189 & 134 & $0.857 \mathrm{D}-02$ & $0.174 \mathrm{D}-03$ \\
16 & 1323 & 136 & $0.368 \mathrm{D}-02$ & $0.812 \mathrm{D}-04$ \\
18 & 1459 & 134 & $0.162 \mathrm{D}-02$ & $0.360 \mathrm{D}-04$ \\
19 & 1593 & 132 & $0.691 \mathrm{D}-03$ & $0.149 \mathrm{D}-04$ \\
\hline 1 & 1725 & 134 & $0.276 \mathrm{D}-03$ & $0.713 \mathrm{D}-05$ \\
\hline & 1859 & 136 & $0.127 \mathrm{D}-03$ & $0.286 \mathrm{D}-05$ \\
\hline 1 & 1995 & 140 & $0.527 \mathrm{D}-04$ & $0.129 \mathrm{D}-05$ \\
\hline
\end{tabular}

Se observa aquí que a partir de IT $=6$ comienza el crecimiento del número de intervalos pero en la décima iteración se detiene esa ten dencia y el valor NINT se estabiliza oscilando alrededor de NINT $=134$. Es importante notar que a pesar de las 38 raíces $y$ aunque $K=70$ es relativamente grande, el número de intervalos parásitos en cada localización no es extremadamente alto. Por ejemplo, en la iteración 21 se obtiene que cada raíz tiene a lo más 6 intervalos asociados y que la mayo ría de ellas poseen 4 o menos; esto quiere decir que para una raíz determinada, en general, no existen más de 3 intervalos parásitos adyacen tes a ella, lo cual es un signo del buen comportamiento del método en el sentido que la convergencia a f del mayorante y minorante no es lenta. 


\section{COMENTARIOS FINALES.}

Aunque no se dijo durante el trabajo, se subentiende que este algoritmo debe ser utilizado con el objeto de realizar una primera apro ximación a las raíces de la función. Una vez que ellas han sido separa das conviene aplicar, por ejemplo, un método del tipo Newton-Raphson para calcularlas con la precisión requerida.

Es claro que los comentarios realizados en sección 3 acerca del método propuesto no pueden generalizarse a todas las funciones puesto que ellos son referidos solamente a los ejemplos en particular ahí consi derados.

\section{REFERENCIAS.}

[1] Basso, P. "Iterative methods for the localization of a global maximum". SIAM J. Numer. Anal. 19 (1982) 4, pp. 781-792.

[2] Basso, P. "Méthodes de localisation du maximum global et des zeros d'une fonction sur un intervalle de la droite numerique". These, Université Scientifique et Médicale de Grenoble, Francia (1978).

[3] Chernousko, F.L. "An optimal algorithm for finding the roots of an approximately computed function" U.S.S.R. Comput. Maths. Phys. 8 (1968) 4; pp. 1-23.

[4] Micchelli,C.A. and Miranker,W.L., "High order search methods for finding roots" J. of Assoc. Comp. Mach. 22 (1975) 1; pp. 51-60.

[5] Sukharev, A.G. "Optimal search for the roots of a function satisfying a Lipschitz condition" U.S.S.R. Comput. Maths. Math. Phys. 16 (1976) 1 ; pp. 17-26. 